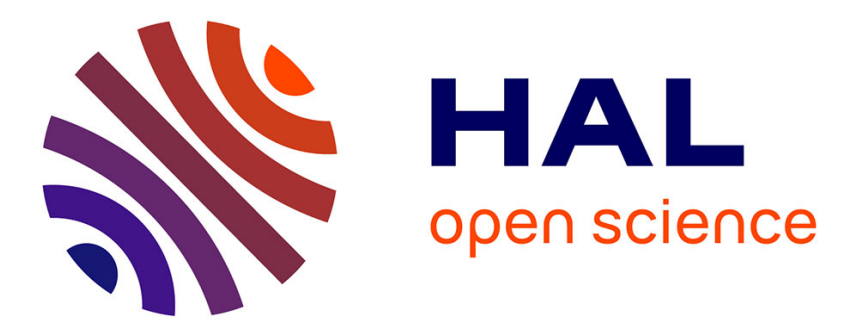

\title{
Low-cost UHF near-field power transmission for RFID applications
}

Marco Fantuzzi, Alessandra Costanzo, Smail Tedjini, Pierre Lemaitre-Auger

\section{To cite this version:}

Marco Fantuzzi, Alessandra Costanzo, Smail Tedjini, Pierre Lemaitre-Auger. Low-cost UHF near-field power transmission for RFID applications. 2017 47th European Microwave Conference (EuMC), Oct 2017, Nuremberg, Germany. pp.212-215, 10.23919/EuMC.2017.8230837 · hal-02388139

\section{HAL Id: hal-02388139 \\ https://hal.univ-grenoble-alpes.fr/hal-02388139}

Submitted on 1 Dec 2019

HAL is a multi-disciplinary open access archive for the deposit and dissemination of scientific research documents, whether they are published or not. The documents may come from teaching and research institutions in France or abroad, or from public or private research centers.
L'archive ouverte pluridisciplinaire HAL, est destinée au dépôt et à la diffusion de documents scientifiques de niveau recherche, publiés ou non, émanant des établissements d'enseignement et de recherche français ou étrangers, des laboratoires publics ou privés. 


\section{Low-Cost UHF Near-Field Power Transmission for RFID Applications}

\author{
Marco Fantuzzi, Alessandra Costanzo \\ Electrical, Electronic, and Information Engineering \\ Department (DEI) \\ University of Bologna \\ Bologna, Italy \\ \{marco.fantuzzi3; alessandra.costanzo\}@unibo.it
}

\author{
Smail Tedjini, Pierre Lemaitre-Auger \\ Grenoble-inp/LCIS \\ University Grenoble Alpes \\ Valence, France \\ \{smail.tedjini; pierre.lemaitre-auger\}@grenoble-inp.fr
}

\begin{abstract}
This paper presents a low-cost contactless power transfer structure for planar configuration working at UHF frequencies. The theory of defected ground structure (DGS) is applied to unconventional materials for power transmission between two closely spaced microstrip lines. Such configuration is studied and described in details, for an easy implementation employing very low-cost materials, characteristics of deemed importance in radio frequency identification (RFID) applications. Influence of nearby material is analyzed and theoretical results are experimentally validated. A possible application for RFID readers is finally proposed.
\end{abstract}

Keywords-wireless power transfer (WPT); near-field; RFID; ultra-high frequency (UHF) communication, defected ground structure (DGS).

\section{INTRODUCTION}

RFID technology is a very well-known technique, which has been deeply investigated since its pervasive distribution in the last decades [1-3]. Its applications span a high number of different scenarios, such as logistics and supply chain, itemlevel inventory tracking, access control and real time location systems (RTLS). A key feature demanded in the vast majority of cases is the necessity for very low-cost materials and manufacturing techniques, both deemed important requirements for the effective mass deployment of RFID technology in market applications [4]. In such a context, the possibility to replace physical connections between hardware components with contactless techniques using inexpensive materials and featuring high repeatability is very attractive and enabling for a practical implementation.

The concept of near-field WPT is extensively studied for different applications, from powering of small devices to wireless charging electric vehicles [5-7]. In almost all cases though, the operating frequency is in the range of the LF or HF, while very few solutions have been proposed at UHF and at microwave frequencies [8-9]. From the RFID point of view, the simplest and less expensive choice would be realizing nearfield coupling at the same frequency of operation of the RFID communication, with no need for additional frequency conversion modules.
Recently a method for near-field coupling at UHF has been proposed [10-11]. This solution exploits the resonant behavior of an optimized DGS to create a near-field coupling between two closely spaced resonators facing each other. This method is here further extended and proposed for low-cost implementation envisioned for RFID applications in the European UHF $868 \mathrm{MHz}$ band. The contactless power transmission between two closely spaced microstrip lines, realized on unconventional low-cost material, is investigated for different application scenarios. Experimental validation of the expected theoretical results is reported.

The paper is organized as follow: in section II, the adopted material and its configuration for the microstrip lines are described; in section III, a brief description of the resonant coupling principles, together with the related parametric investigations, are introduced. Results for quasi real-scenario implementations are provided in section IV, while, in section $\mathrm{V}$, a possible application for RFID readers is proposed. Finally, the main results obtained are summarized in the conclusions.

\section{Material Choice AND Chosen Substrate}

One of the most challenging requirements of RFID systems is a low-cost implementation. A second feature that is of increasing importance in many emerging applications is a flexibility of the structure, in order to be conformable with nonplanar surfaces. The choice of material should be based on

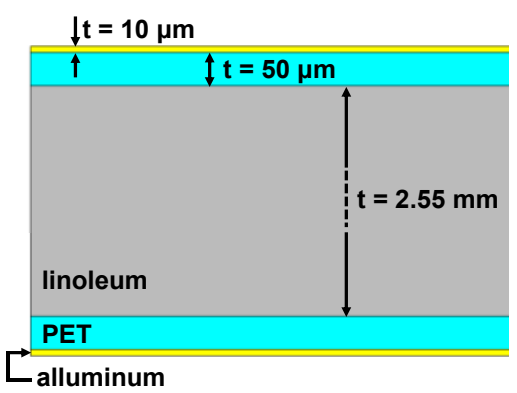

Fig. 1. Multilayer substrate representation. Two PET layers with printed conductive aluminum are employed as top and bottom metallization. 
these two requirements. For this purpose thin PET, which is flexible, low-cost and widely adopted in RFID systems especially for the tags realization, is a good candidate. Nevertheless, it is suitable as a supporting material for printing antennas, but it is usually too thin to be adopted as the sole substrate for microstrip lines and antennas. In order to provide the convenient thickness, linoleum, a very cheap material widely used for floor covering, tough not directly suitable for $\mathrm{RF}$ applications, can be adopted as a further supporting medium besides the PET. The combination of these two materials offers a very cheap solution that can be effectively adopted for large-scale applications. The electrical properties of the two materials are measured by means of a standard resonant cavity method and are found to be $\varepsilon_{r}=3.2, \tan (\delta)=$ $0.003(@ 1 \mathrm{GHz})$ and $\varepsilon_{r}=1.89, \tan (\delta)=0.011(@ 1 \mathrm{GHz})$ for PET and linoleum, respectively. A schematic representation of a possible multilayer low-cost substrate adopted for the present design is reported in Fig. 1. Printed aluminum on PET is employed for the metallic layers. The three layers can be simply superimposed on top of each other, without any extra glue or fixing material.

\section{CONTACTLESS Resonator Design}

As described in [11], the resonant coupling circuit can be obtained by a proper design of an $\mathrm{H}$-shaped DGS, which can be modelled as a parallel LC resonator, where the inductive contribution comes from the two rectangular removals in the ground plane and the capacitive one is realized by introducing a lumped SMD capacitor that provides the needed value of capacitance. In the present design, SMD components are avoided and an interdigital capacitor is adopted to simplify the manufacturing and to lower the overall cost. Therefore, all metallic structures are planar and can be directly printed on a PET substrate.

For an effective implementation in real scenarios, the evaluation of the impact of surrounding materials which are

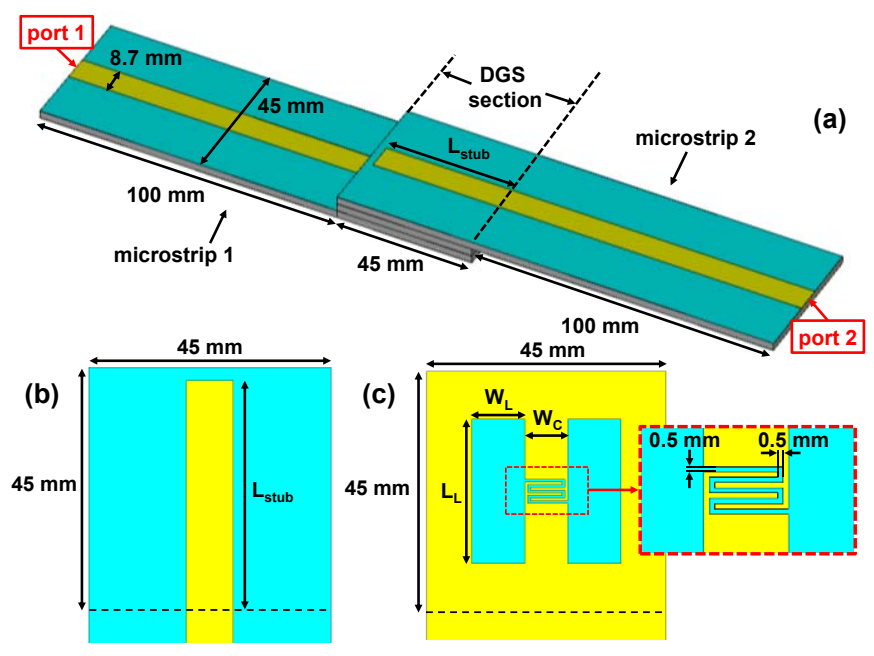

Fig. 2. Representation of the proposed structure and relative dimensions. (a) Perspective view of the two microstrip lines ending/beginning with the DGS section; one layer of linoleum is placed as spacer between the two DGSs. (b) Top view of the DGS section. (c) Bottom view of the DGS section.

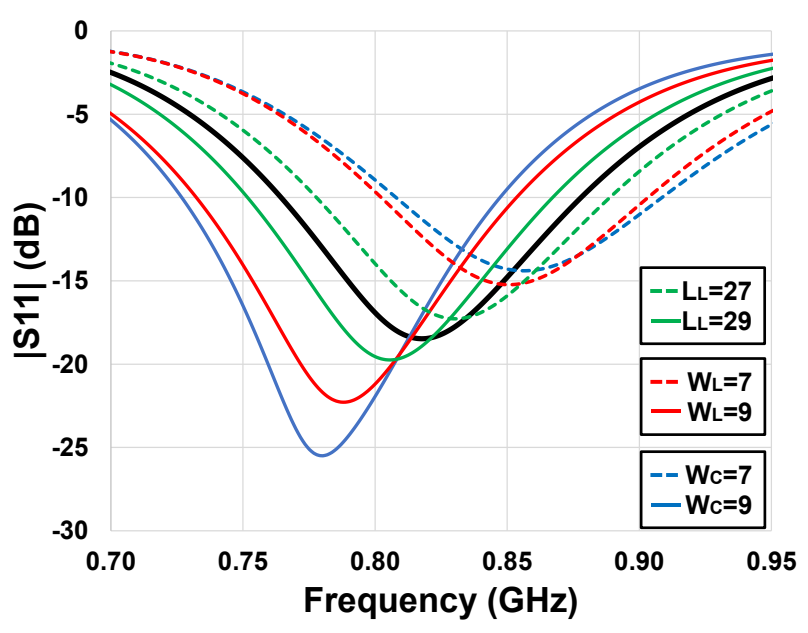

Fig. 3. Shift in the resonant frequency obtained by varying the significant parameters of the DGS. The black curve represents the reference starting point, obtained for $L_{L}=28 \mathrm{~mm}, W_{L}=8 \mathrm{~mm}$ and $W_{C}=8 \mathrm{~mm}$.

placed nearby the resonating structure are carried out. The strip line of the structure reported in [11] is facing the supporting material. So, placing the whole structure onto any material other than air will deeply affect its behavior. Indeed, either the effective dielectric constant of the bottom microstrip will change or it will become a stripline if it is placed on top of a metallic surface. To overcome this limitation, the bottom microstrip line is flip over such that the ground plane is facing the supporting material. The effect of the latter is restricted to the area of the DGS due to the partial metal removals in the ground plane. This way, the effect of different materials used as support has to be evaluated only in this region.

The multilayer substrate described in the previous section is chosen for the investigation of the near-field coupling performance between the two closely spaced resonators. The proposed structure is depicted in Fig. 2, along with the corresponding dimensions. By tuning the dimensions of the structure, a parametric study is conducted with electromagnetic (EM) simulations in order to provide the desired coupling behavior at the European $868 \mathrm{MHz}$ RFID band. As a first step, the dimensions of the interdigital capacitor are found in order to provide a fixed capacitance of approximately $1 \mathrm{pF}$ [12]. Secondly, the other dimensions reported in Fig. 2 are tuned in order to highlight their impact on the inductive behavior of the DGS and therefore on the operating frequency. Fig. 3 illustrates some results reporting the impact of the parameters $L_{L}, W_{L}$ and $W_{C}$ on the resonant frequency. One parameter is changed at a time while keeping the other two fixed. From these results, it is possible to notice that $L_{L}$ has a smaller impact, it produces a shift of approximately $10-15 \mathrm{MHz} / \mathrm{mm}$, and it is therefore suitable for fine tuning of the resonant frequency. $W_{L}$ and $W_{C}$ have a greater influence, introducing shifts of about $35-40 \mathrm{MHz} / \mathrm{mm}$. The modification of $W_{L}$ is preferred since the latter parameter influences also the interdigital capacitor. The length of the stubs, $L_{s t u b}$, is kept equal to $20 \mathrm{~mm}$.

The second significant design parameter is the distance between the two resonators. It is well known in near-field 

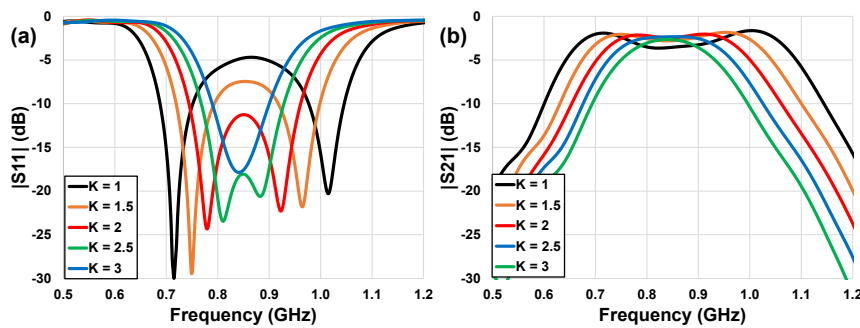

Fig. 4. Effect of the number of linoleum layers, $K$. DGS dimensions are $L_{L}=27 \mathrm{~mm}, W_{L}=8 \mathrm{~mm}, W_{C}=8 \mathrm{~mm}$ and $L_{s t u b}=18 \mathrm{~mm}$. (a) Reflection coefficient. (b) Transmission coefficients.

resonant WPT applications that moving the two resonators from the optimal distance produces a frequency separation of the two resonances [13]. Such phenomenon is graphically described in Fig. 4, where the simulated performance of the near-field coupling is reported for different values of distance. For a real physical implementation of the structure, the material used as spacer between the two resonators cannot simply be free space but necessarily has to be a physical medium. Hence, to comply with the low-cost requirements, the same linoleum material already used is employed as spacer.

It is interesting to notice from the results of Fig. 4 that, even though the optimum distance provides the best operating bandwidth, the maximum transmission value is obtained for $K=1$, which is therefore chosen for the present design. The 0.5 $1 \mathrm{~dB}$ drop of the transmission coefficient for higher values of $K$ can be explained by the increased quantity of lossy materials inserted between the two resonators. Even if it is reduced, the operating bandwidth obtained is still well suitable for applications in the European $868 \mathrm{MHz}$ band. Obviously, in case a higher bandwidth would be required, for example for coverage of other RFID frequency bands, the optimum distance could be chosen, at the expense of a slight reduction of the transmission coefficient.

\section{IMPLEMENTATION AND MEASURED RESULTS}

Based on the previously described considerations, two prototypes of the proposed system were realized and measured. For a practical implementation, it is extremely important to evaluate the effect of the materials surrounding the DGS section, specifically the physical support on which the coupling structure is placed during operation. In this section, the effects of plastic and wood materials are first considered as they have the same influence on the coupling structure. In a second step, metallic material is also assessed, estimated as the most influencing yet widely adopted medium for a vast number of applications. The pictures of the two measurement setups are reported in Fig. 5. In both cases, a single additional layer of linoleum is placed between the coupling structure and the physical support.

\section{A. Non-metallic Environmenet}

The dimensions of the DGS are first optimized for the structure to be put over a plastic or wood material. For that, the first of the two resonances is shifted in the UHF band. The values of the parameters are $L_{L}=26 \mathrm{~mm}, W_{L}=6 \mathrm{~mm}, W_{C}=7$ $\mathrm{mm}$ and $L_{s t u b}=18 \mathrm{~mm}$. Fig. 6 reports the behavior of the

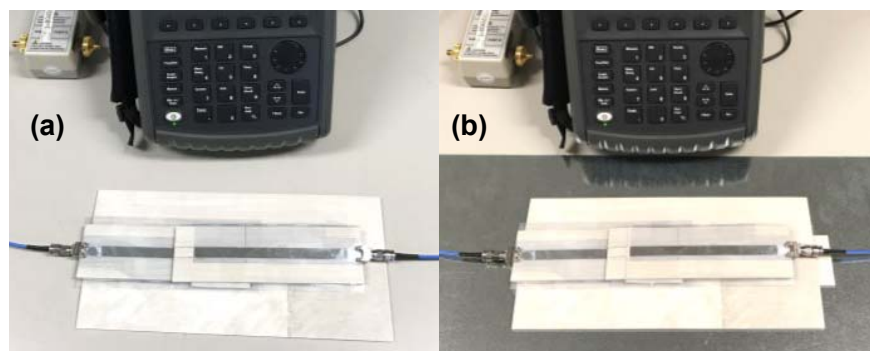

Fig. 5. Pictures of the measurement setup placed on different material. (a) Non-metallic support. (b) Metallic support.

structure in terms of reflection and transmission coefficients. A good agreement is observed between measured and simulated data. The slight discrepancies are mainly due to realization tolerances and to small airgaps inevitably present between the different layers of the adopted substrate, depicted in Fig. 1. Moreover, it is worth noting that a slight influence on the performance is also introduced by the bending of the second microstrip line, because of the thickness of the linoleum layer introduced as spacer between the two resonators.

\section{B. Metallic Environment}

In a large number of applications, the RFID operation has to take place in close proximity of metallic objects. This represents by far the worst possible scenario and it is therefore chosen as the second case study. Once again, the dimensions of the structure were optimized by EM simulations. It is found that placing the resonating structure on top of a metallic surface exerts a significant influence on the behavior of the coupling. In particular, the presence of the metallic surface appears to have a detrimental effect mainly on the first of the two resonances of Fig. 6, canceling it, while the second resonance is only slightly affected. This effect is explained by the fact that the first resonance is mainly attributed to the DGS closer to the metallic surface. The second resonance, conversely, mainly attributed to the further DGS, features only a slight frequency shift and a small degradation in the transmission coefficient. It is therefore chosen for the present design. A second prototype was realized by shifting the frequency of the second resonance

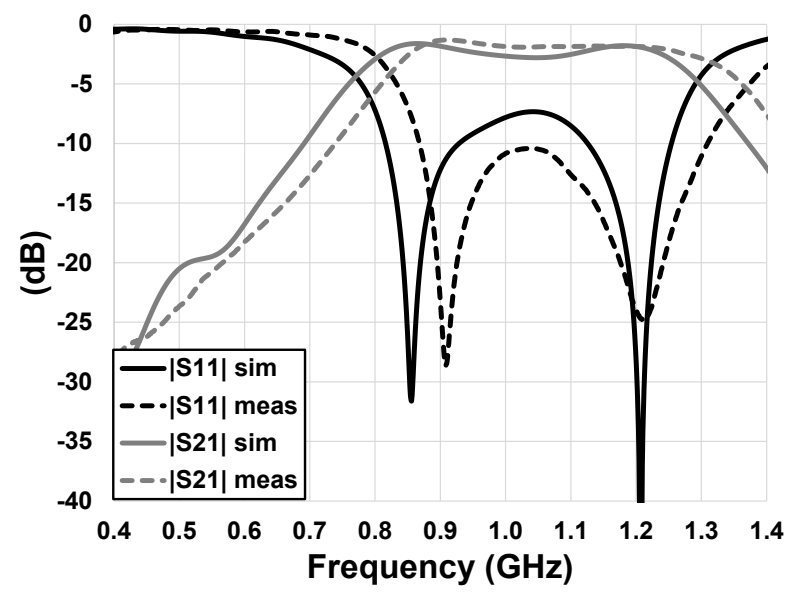

Fig. 6. Comparison between simulated and measured reflection and transmission coefficients for non-metallic environment. 


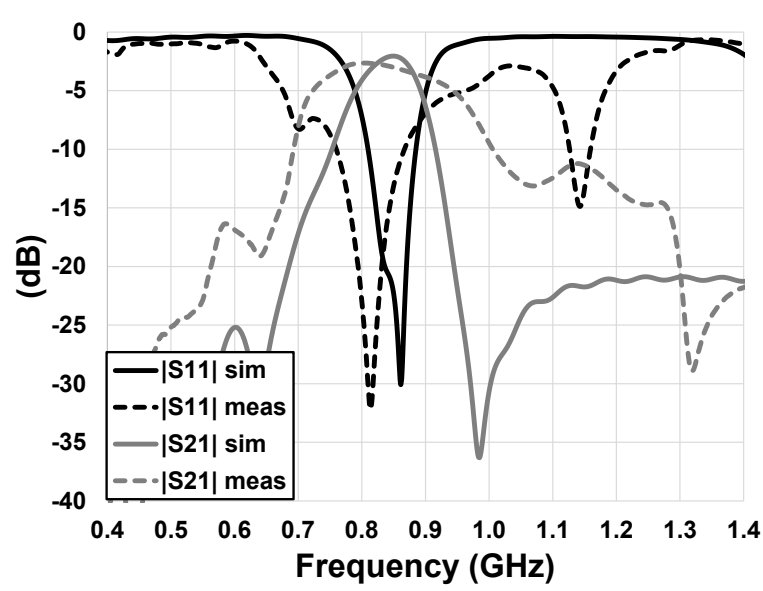

Fig. 7. Comparison between simulated and measured reflection and transmission coefficients for metallic environment.

to be centered at $868 \mathrm{MHz}$. The corresponding dimensions are in this case: $L_{L}=27 \mathrm{~mm}, W_{L}=10 \mathrm{~mm}, W_{C}=11 \mathrm{~mm}$ and $L_{s t u b}=$ $14 \mathrm{~mm}$. Comparison between the measured and simulated data is provided in Fig. 7.

\section{RFID READER APPLICATION}

The present design can be applied to a large number of applications, where the possibility of providing contactless coupling between two planar microstrip lines that need to operate at UHF sounds attractive. A possible implementation can be that of wireless connection between an RFID reader unit and its antenna. Indeed, there are a several number of cases in which the radiating element of an RFID reader needs to be replaced or modified in a certain way. Since these kinds of operations typically involve a very high number of units at a time, a contactless connection can result in a significant cost reduction with respect to a solution requiring physical connections. Moreover, its high repeatability can easily speed up the entire process.

As a proof of concept, the microstrip line connected to the second DGS, terminated by port 2 of Fig. 2, is connected to a patch antenna, designed on the same multilayer substrate reported in Fig. 1. The designed patch antenna is fed with an inset of $34 \mathrm{~mm}$ for impedance matching and it has a width and length of 145 and $122 \mathrm{~mm}$ respectively. The dimensions of the DGS are the same as those employed for the prototype of section IV-A, except for the stubs which are in this case tuned to different lengths for matching purposes, since the loads of the two DGSs are different in the present case. In particular, the length of the stub fed by port 1 is fixed to $16 \mathrm{~mm}$, while the length of the second stub is chosen equal to $20 \mathrm{~mm}$. The simulated performance of the system show a maximum realized gain equal to $4.9 \mathrm{dBi}$ and a radiation efficiency of $45 \%$, values mainly limited by the lossy substrate employed.

\section{CONCLUSIONS}

A novel low-cost implementation for contactless near-field coupling between two resonating structure at UHF is demonstrated for European $868 \mathrm{MHz}$ RFID applications. The effects of different nearby low-cost materials used as support for the resonant structure were evaluated and the EM design was confirmed by measurement results. The substrate used in the present study entitles the proposed system for wide deployment in mass market applications thanks to its very low cost and high repeatability. Finally, a possible application envisioned for antenna wireless connection in RFID readers is proposed.

\section{ACKNOWLEDGMENT}

This work was partially supported by the COST Action IC1301 - Wireless Power Transmission for Sustainable Electronics (WiPE).

\section{REFERENCES}

[1] K. V. S. Rao, P. V. Nikitin, and S. F. Lam, "Antenna design for UHF RFID tags: a review and a practical application," IEEE Transactions on Antennas and Propagation, vol. 53, no. 12, pp. 3870-3876, Dec. 2005.

[2] G. Marrocco, "The art of UHF RFID antenna design: impedancematching and size-reduction techniques," IEEE Antennas and Propagation Magazine, vol. 50, no. 1, pp. 66-79, Feb. 2008.

[3] E. Perret, S. Tedjini, and R. S. Nair, "Design of Antennas for UHF RFID Tags," Proceedings of the IEEE, vol. 100, no. 7, pp. 2330-2340, July 2012 .

[4] F. Lolli, M. Virili, G. Orecchini, M. Dionigi, F. Alimenti, P. Mezzanotte, and L. Roselli, "Electromagnetic Characterization of Paper-Glue Compound for System-in-Package on Paper (SiPoP) Future Developments," IEEE Microwave and Wireless Components Letters, vol. 22, no. 10, pp. 545-547, Oct. 2012.

[5] B. L. Cannon, J. F. Hoburg, D. D. Stancil, and S. C. Goldstein, "Magnetic Resonant Coupling As a Potential Means for Wireless Power Transfer to Multiple Small Receivers," IEEE Transactions on Power Electronics, vol. 24, no. 7, pp. 1819-1825, July 2009.

[6] Z. N. Low, R. A. Chinga, R. Tseng, and J. Lin, "Design and Test of a High-Power High-Efficiency Loosely Coupled Planar Wireless Power Transfer System," IEEE Transactions on Industrial Electronics, vol. 56, no. 5, pp. 1801-1812, May 2009.

[7] J. Shin et al., "Design and Implementation of Shaped MagneticResonance-Based Wireless Power Transfer System for RoadwayPowered Moving Electric Vehicles," IEEE Transactions on Industrial Electronics, vol. 61, no. 3, pp. 1179-1192, March 2014.

[8] R. Trevisan and A. Costanzo, "A UHF Near-Field Link for Passive Sensing in Industrial Wireless Power Transfer Systems," IEEE Transactions on Microwave Theory and Techniques, vol. 64, no. 5, pp. 1634-1643, May 2016.

[9] S. Kim, J. S. Ho, and A. S. Y. Poon, "Wireless Power Transfer to Miniature Implants: Transmitter Optimization," IEEE Transactions on Antennas and Propagation, vol. 60, no. 10, pp. 4838-4845, Oct. 2012.

[10] S. Hekal, A. B. Abdel-Rahman, H. Jia, A. Allam, R. K. Pokharel, and H. Kanaya, "Strong resonant coupling for short-range wireless power transfer applications using defected ground structures," IEEE Wireless Power Transfer Conference (WPTC), Boulder, CO, 2015, pp. 1-4.

[11] S. Hekal, A. B. Abdel-Rahman, H. Jia, A. Allam, A. Barakat, and R. K. Pokharel, "A Novel Technique for Compact Size Wireless Power Transfer Applications Using Defected Ground Structures," IEEE Transactions on Microwave Theory and Techniques, vol. 65, no. 2, pp. 591-599, Feb. 2017.

[12] W. Withayachumnankul, C. Fumeaux, and D. Abbott, "Compact electric-LC resonators for metamaterials," Optics Express, vol. 18, no. $25,25912-25921,2010$

[13] A. Costanzo, M. Dionigi, F. Mastri, and M. Mongiardo, "Rigorous modeling of mid-range wireless power transfer systems based on royer oscillators," IEEE Wireless Power Transfer Conference (WPTC), Perugia, 2013, pp. 69-72. 